aimed to investigate the characteristics of false negative pulmonary embolism cases by $\mathrm{V} / \mathrm{Q}$ scan.

Method During Jun 2008 to Apr 2010, patients with acute pulmonary embolism underwent both ventilation-perfusion scintigraphy $(\mathrm{V} / \mathrm{O}$ scan) and spiral CT pulmonary angiography (CTPA) were systematically reviewed. The patients were grouped by results of CTPA. Group 1: CTPA showed that the main pulmonary or lobe arteries were involved; Group 2: CTPA showed that the thrombi were limited to segmental or subsegmental pulmonary arteries. The characteristics of the false negative cases by V/O scan were analysed.

Results In all 35 acute pulmonary embolism patients were included. There were 13 males (37.1\%) and 22 females (62.9\%) with a mean age of $59.3 \pm 15.0$ years. The mean onset time of pulmonary embolism was $9.9 \pm 7.3$ days and the mean interval time between $\mathrm{V} / \mathrm{O}$ scan and CTPA was $3.7 \pm 2.5$ days. There were 1 (4.4\%) false negative cases out of 23 patients by $\mathrm{V} / \mathrm{O}$ scan in group 1 and $4(33.3 \%)$ false negative cases out of 12 patients in group $2(p<0.001)$.

Conclusions The incidence of false negative cases in diagnosing acute pulmonary embolism by $\mathrm{V} / \mathrm{O}$ scan is increased significantly when CTPA showed that thrombi are limited to segmental or subsegmental and further branches of pulmonary arteries. In the clinical setting of highly suspected acute pulmonary embolism, even though the $\mathrm{V} / \mathrm{Q}$ scan was negative, CTPA is needed for more diagnostic information.

\section{e0578 DIVERSITY OF PLATELET INHIBITION UNDER CLOPIDOGREL AND ASPIRIN MEASURED BY VARIOUS ASSAYS}

doi:10.1136/hrt.2010.208967.578

Li Jian, Cong Yulong, Dong Wei, Wang Chengbin, Zhang Huawei. Chinese Pla General Hospital

Objective Characterize the inhibition of platelet P2Y12 receptor and COX-1 pathway after clopidogrel adding to aspirin intake.

Methods 32 inpatients with coronary atherosclerosis were enrolled; $600 \mathrm{mg}$ clopidogrel was given in consecutive two days, $100 \mathrm{mg} / \mathrm{d}$ Aspirin intake simultaneously. Inhibition of platelet aggregation induced by $\mathrm{ADP} / \mathrm{AA}$ on Thrombelastography, platelet aggregation activated by $\mathrm{ADP} / \mathrm{AA}, \mathrm{CD} 62 \mathrm{p}$ and vasodilator stimulated phosphoprotein (VASP) were measured at no clopidogrel, 10th hour and 36 th hour after the first $300 \mathrm{mg}$ loading dose of clopidogrel.

Results (1) Inhibition ADP increases to $(42.5 \pm 29.1) \%$ statistically $(p=0.034)$ at $10 \mathrm{~h}$ but not continues to the 36 th hour $(p=0.106)$, Inhibition $\mathrm{AA}$ increases from $(56.6 \pm 36.6) \%$ to $(85.4 \pm 20.8) \%$ statistically $(p=0.101)$ at $36 \mathrm{~h}$, indicates COX-1 pathway is inhibited stronger than single aspirin intake. (2) Aggregation ADP decreases statistically ( $\mathrm{p}=0.036$ ) until $36 \mathrm{~h}$, Aggregation AA decrease statistically $(p=0.021)$ at $10 \mathrm{~h}$ and stabled to $36 \mathrm{~h}(\mathrm{p}=0.045)$. Platelet response described by aggregation is different from inhibition percentage on TEG. (3) Change of Platelet Reactivity Index (PRI) in VASP assay is similar to Aggregation ADP, CD62p fluctuates from $(7.5 \pm 1.4) \%$ to $(4.2 \pm 1.1) \%(p=0.035)$ and $(4.3 \pm 0.2) \%(p=0.211)$ in a different way. Conclusion No correlation could be found between results of platelet inhibition and aggregation, induced by whether ADP or AA. VASP helps to identify platelet responses to Clopidogrel specific. Absence of standard on platelet function measurement results in variety of clopidogrel resistance study.

\section{e0579 NOVEL COAGULATION REgIME METHOD FOR CTO PRIOR TO PCI}

doi:10.1136/hrt.2010.208967.579

Michael CL Lim. Singapore Medical Specialists Centre

Objective The aim of this study is to demonstrate that coagulation regime for patients with CTOs enable successful PCI.
Methods During the period of 2009-2010, a total of six patients had undergone CT Coronary Angiogram and were found to have long CTO (more than $3 \mathrm{~cm}$ ) in a single vessel. These patients had undergone prior PCI for the CTOs which were unsuccessful; the CTO could not be crossed. The patients were prescribed Warfarin for 6 months to a year. Their INRs were titrated to 1.5-2.0.

Results All six patients underwent successful PCI performed by a competent operator with more than 30 years experience and had performed more than $1000 \mathrm{PCI}$ cases. There was no MACE or complications encountered with the complex CTO lesions.

Conclusions This pilot study illustrates the safety and feasibility of giving anticoagulation prior to PCI for complex CTO lesions.

\section{Clinical and Research Medicine: Hypertension e0580 COMBINED NIFEDIPINE SUSTAINEDRELEASE TABLET WITH BETALOC TO TREAT ESSENTIAL HYPERTENSION}

doi:10.1136/hrt.2010.208967.580

${ }^{1}$ Ding Shaoxiang, ${ }^{2} \mathrm{Hu}$ Kunfang, ${ }^{2}$ Wang Wei. ${ }^{1}$ Kang Le Hospital, Qinghai Province, China; ${ }^{2}$ Center of Disease Control, Middle District Of Xining, China

Objective To investigate the clinical efficacy of the treatment of essential hypertension combined nifedipine sustained-release tablets with betaloc.

Methods 60 cases of essential hypertension discovered recently were divided into two groups in random, 30 patients in each group. The control group were given nifedipine sustained-release tablets $10 \mathrm{mg}$, twice a day at 08:00 and 20:00 oral; treated group were at the same of nifedipine sustained-release tablets with control group, but increased betaloc $25 \mathrm{mg}$ before lunch. Measured blood pressure twice a week and total of 8 weeks.

Results In control group, significant therapeutic effect in 11 cases, there were clear therapeutic effect in 11 cases, no treatment effect in 8 cases, the total therapeutic efficacy was 73.3\%; In treated group, significant therapeutic effect in 18 cases, there were clear therapeutic effect in 11 cases, no treatment effect in 1 cases, the total therapeutic efficacy was $96.7 \%$; There was significant difference between two groups $\left(\mathrm{X}^{2}=7.13, \mathrm{p}<0.05\right)$. In control group, there was no significant change in heart rate $(p>0.05)$; But in treatment group, heart rate decreased at average of 10 times $\mathrm{min}^{-1}$, There was obvious difference in Statistics $(\mathrm{p}<0.05)$.

Conclusions It is a good way to treat essential hypertension combined nifedipine sustained-release tablets with betaloc, and there is a mutual synergy, worthy to be popularised.

\section{E0581 EFFECT OF ECG CHARACTERISTIC AND CLINICAL PROGNOSIS OF THE VENTRICULAR ELECTRICAL STORM IN PATIENTS WITH HYPERTROPHY AND ACUTE MYOCARDIAL INFARCTION}

doi:10.1136/hrt.2010.208967.581

Yu Wenjiang. Laboratory of Electrocardiography, Yantai Yuhuangding Hospital, Yatai, China

Objective To investigate the effect of ECG characteristic and clinical prognosis of ventricular electrical storm (VES) in patients with hypertrophy and acute myocardial infarction (AMI).

Methods 317 cases of VES in patients with hypertrophy and AMI group. Sixty cases of after AMI patients non-VES group. The analysed of ECG examination and keep watch on ECG was relationship between clinical features and prognosis among the two groups.

Results In VES group PTFv1 abnormal, $\sum$ ST segment elevation amplitude, ST segment elevation leads, ST segment reduction 